\title{
Arbeit mit inneren Anteilen
}

\section{Kristina Scheuffgen · Falko von Ameln}

Online publiziert: 14. Februar 2019

(C) Springer Fachmedien Wiesbaden GmbH, ein Teil von Springer Nature 2019

Liebe LeserInnen,

nach dem zuletzt veröffentlichten Heft zum Thema „Integrationsphase“ kommt nun ein Heft zu einem Thema, welches aktuell in der Psychotherapie- und Beratungsszene vielfältig in Erscheinung tritt sowie auch Popularität im gesamtgesellschaftlichen Kontext erreicht hat (siehe zum Beispiel der Kinofilm „Inside Out“1). Innere Anteile sind en vogue, erscheinen zum Beispiel in Form der Ego State-Therapie, der Schematherapie, der systemischen Aufstellungsarbeit, in unterschiedlichen Settings wie Psychotherapie, Coaching und Supervision. Es ist ein bisschen gewagt sich diesem Thema zu widmen, da es auf der einen Seite zurzeit so inflationär und Schulen übergreifend verwendet wird und andererseits für uns als PsychodramatikerInnen ursprünglich in unserem Verständnis des Selbst verankert ist. So ist der Begriff der inneren Anteile in der Definition des Selbst, welches nach Moreno aus einer Vielzahl von Rollen besteht, enthalten. Es ist nach Moreno gerade die Fähigkeit des Menschen situativ unterschiedliche Rollen einzunehmen und zu „spielen“, die es uns erlaubt, spontan und kreativ zu handeln.

Innere Anteile können verborgen oder sichtbar, fremd oder eigen, dissoziiert oder integriert, traumatisiert oder gesund, gesteuert oder ungesteuert, jung oder alt, bzw. kindlich oder erwachsen und noch vieles mehr sein. Störungsspezifisch betrachtet können sie durch einen Bewältigungsversuch im Umgang mit traumatisierenden Erfahrungen entstehen. Im Hinblick auf die Persönlichkeitsentwicklung insgesamt

\footnotetext{
1 Zu Deutsch: „Alles steht Kopf“. Disney Pixar Animation Film aus dem Jahr 2015, in dem Emotionen als innere Rollen verkörpert werden.
}

F. von Ameln $(\bowtie)$

Am Diekschloot 11b, 26506 Norden, Deutschland

E-Mail: info@vonameln.net 
können sie als die Vielzahl von Rollenrepräsentationen, die im Rahmen der individuellen Sozialisation in Familie, Beruf, Hobby, Nationalität und Globalität entstehen, konzeptionalisiert werden.

Wenn wir von inneren Anteilen sprechen ist es unweigerlich wichtig, dass diese nicht einfach nur da sind, sondern dass wir diese wahrnehmen können, Verbindungen schaffen können und damit in die Lage versetzt werden, Veränderungen vorzunehmen. Die Wahrnehmung unserer inneren Anteile erfordert eine Distanz zu diesen. Wer ist es denn dann, der diese betrachten kann? Welcher innere Anteil übernimmt die Selbstreflektion? Welche Anteile kann dieser Anteil betrachten? Bleibt als innerer Anteil immer so etwas wie ein dissoziierter, beobachtender Selbstanteil, der uns die Mentalisierung unserer Erfahrungen ermöglicht? In jedem Fall bleibt es unsere menschliche Fähigkeit von außen auf uns selbst zu schauen, uns unserer selbst bewusst zu sein, und dies differenziert und pluralistisch. Eine solche Vorstellung kann jedoch auch Ängste vor Fragmentierung und Identitätsverlust auslösen. Umso versöhnlicher ist die Vorstellung, dass es ja darum geht, sich selbst kennen und verstehen zu lernen, die Handlungskompetenzen dadurch zu erweitern, dass unsere Inneren Anteile sich miteinander verbinden und integrieren lassen, wenn wir es denn wünschen.

Wir leben im Kontinuum der Zeit, linear mit Terminen, Routinen, Abfolgen, Planungen, Vereinbarungen, Ergebnissen, Regeln etc. Unser Sein ist aber umfänglicher, Vergangenheit, Gegenwart und Zukunft sind in unserer Bewusstheit und Erfahrung enthalten. Um präsent zu sein, mit uns selbst und mit anderen, müssen wir einen Aufmerksamkeitsfokus wählen, permanent Entscheidungen treffen, und kommunizieren bewusst oder unbewusst miteinander und mit uns selbst. Zunehmend finden sich Hinweise darauf, dass unsere bewussten neokortikalen Prozesse im Grunde durch viel schnellere vorsprachliche Wahrnehmungsprozesse der subkortikalen Hirnprozesse gesteuert werden (siehe z. B. Embodiment, Embodied Cognition). So kommt unserer zum Teil unbewussten Intuition im Gegensatz zur „Selbst“-Bewusstheit zunehmend mehr Bedeutung zu. Diese Konzepte bestätigen unser psychodramatisches Verständnis darüber, dass tiefgreifende Veränderungsprozesse über ein interaktives, bewegtes Erleben geschehen. Es ist deshalb so spannend, den eigenen inneren Anteilen auf die Spur zu kommen, da sie zum Teil enorme Wirkungs- und Steuerungskraft haben, die uns zumindest situativ immer wieder verborgen bleibt und uns gerade deshalb handlungsfähig bleiben lässt. So ist es ist immer nur ein Ausschnitt von uns, den wir in den festgelegten Dimensionen von Zeit und Raum uns selbst und anderen präsentieren können. In unserem psychodramatischen Verständnis können wir diese Limitierung durch die Surplus-Realität aufheben, das „,wahre zweite Mal“ auf der Bühne inszenieren und wir können spontan und kreativ nicht nur unserer Innenwelt auf der Bühne Gestalt geben (inner world outside), sondern auch unser Selbst pluralisieren, die Singularität verlassen und unsere inneren Anteile auf die Bühne bringen (inner parts outside). Wie wir das Ganze nun nennen, ob wir von inneren Anteilen, inneren Rollen, Ego States, Ich-Anteilen, dem inneren Team, Introjekten, Selbstund Objektrepräsentanzen oder inneren Stimmen sprechen, ist dabei unserer jeweiligen Schule und aktuellem Fokus geschuldet. Letztlich geht es darum, uns selbst zu verstehen und dabei benötigen wir zur Innenschau (der Sicht auf unsere inneren Anteile) das Außen (den Spiegel, die mentalisierte Position). Unserer Kreativität ist 
es zu verdanken, dass der Umgang mit uns selbst freier werden kann, wir wählen dürfen, individuelle Entwicklungen vornehmen können, letztlich um unsere Selbstaktualisierung zu fördern. Welch privilegierte Position, dass wir uns mit unseren inneren Anteilen auseinander setzen können und dürfen und gleichzeitig so wichtig in dieser Welt, in der fixierte, faschistische Glaubenssätze wieder mehr Zuspruch und Verbreitung erfahren. Bei einer Vorstellung unseres Selbst als einer Ansammlung von Rollen oder inneren Anteilen steigt auch unsere Verbundenheit zueinander, da wir dies in Form von unseren diversen kulturellen Atomen miteinander teilen. Dadurch wird es schwerer, rigide Pseudoabgrenzungen oder willkürliche Kategorien von Gender, Rasse oder Nationalität aufrechtzuerhalten. Ein Handeln im Bewusstsein unserer inneren Anteile setzt eine mentalisierte Position voraus, die letztlich eine Transzendenz von Festlegung, Restriktion, Singularität und Rollenkonserve ermöglicht.

In dem vorliegenden Heft gestaltete sich die Suche nach AutorInnen für die Grundlagenartikel nicht ganz einfach. So ist es umso erfreulicher, dass aus einem angedachten Grundlagenartikel plötzlich zwei entstanden sind. Vielleicht ist es deshalb ebenso dazu gekommen, dass wir nun letztlich vier englischsprachige Beiträge in diesem Heft veröffentlichen, einer davon international und themenspezifisch aus dem Bereich der psychodramatischen Traumatherapie.

Im Thementeil haben wir somit zum einen einen differenzierten Grundlagenartikel, in dem es gelungen ist, aktuelle und relevante theoretische Konzepte der Psychologie mit theoretischen psychodramatischen Konzepten zu einem umfassenden Verständnis von inneren Anteilen integrierend zu vermitteln. Dieser Grundlagenartikel von Konrad Schnabel, Falko von Ameln und Christian Stadler nähert sich dem Thema der inneren Anteile aus einer theoretischen Perspektive verschiedener Ansätze. Ausgehend von Morenos Persönlichkeitstheorie sowie vom Modell des Inneren Teams (Schulz von Thun 1998) wird ein pluralistisches Bild des Selbst mit Erkenntnissen aus persönlichkeitspsychologischer, entwicklungspsychologischer und sozialpsychologischer Perspektive angereichert und aus dieser aktuellen Fachlichkeit werden Konsequenzen für die psychodramatische Praxis abgeleitet. Es werden vier Thesen aufgestellt, die Morenos hochmodernen Begriff der Rolle mit dem heute allgemeiner und schulenübergreifend benutzten Begriff der inneren Anteile verbinden und es wird die Praktikabilität dieser Konzepte für Therapie und Beratung deutlich gemacht.

Ein zweiter, dem Psychodrama als Verfahren gewidmeter Grundlagenbeitrag von Falko von Ameln, Konrad Schnabel und Christian Stadler präsentiert einen Orientierungsrahmen für eben diese Arbeit mit inneren Anteilen. In diesem Beitrag erhalten die LeserInnen einen praxisnahen, konkreten Ablauf für diese psychodramatische Arbeit. Den Autoren ist eine verständliche, theorie- und zielgeleitete Darstellung der erforderlichen, einzelnen Handlungsschritte für die Leitung in der Arbeit mit inneren Anteilen gelungen. Dabei geht es in der Anwendung nicht spezifisch um den Einsatz der Methode bei traumabedingten inneren Anteilen, sondern generell um die psychodramatische Arbeit in dem Verständnis des Selbst als einer Ansammlung von Rollenrepräsentationen bzw. inneren Anteilen, geltend für alle Menschen. Ein Augenmerk wird auf die erforderlichen Handlungskompetenzen der PsychodramaleiterInnen gelegt. 
Lisa Tomaschek-Habrina stellt in ihrem Beitrag die Arbeit mit inneren Anteilen aus dem Bereich des Coachings vor. Der innere Dialog wird hier als psychodramatisches Instrument in Form von drei unterschiedlichen psychodramatischen Arrangements vorgestellt: dem Soliloquium (Selbstgespräch auf der Mikrobühne), dem Metalog (Dialog in der 3. Person auf der Raumbühne) und dem soziokulturellen Atom (mit Intermediärobjekten auf der Tischbühne). Es geht jeweils darum, die inneren Anteile zu externalisieren und zu personalisieren. Jedes Arrangement wird anhand eines Fallbeispiels strukturiert veranschaulicht. Ziel ist es, die Selbstwahrnehmung zu differenzieren und damit die Handlungsmöglichkeiten mit sich selbst (und seinen Selbstanteilen) und anderen (und deren Selbstanteilen) zu erweitern und zu präzisieren. Die Technik des psychodramatischen Rollenwechsels ist hierbei die zentrale Technik bei allen drei vorgestellten Arrangements.

Wir haben einen englischsprachigen Beitrag aus den USA von Kate Hudgins, die ein eigenes Modell zur Arbeit mit Inneren Anteilen aus dem Bereich der Traumatherapie entwickelt hat, das Therapeutic Spiral Model (TSM). Hudgins entwickelte dieses Modell zur therapeutischen Arbeit mit inneren Anteilen bei traumatisierten Menschen bereits 1992 und hat seitdem internationale Weiterbildungen in mehr als 35 Ländern dazu gegeben sowie Wirksamkeitsstudien diesbezüglich unternommen. Sie gibt uns zunächst einen theoretisch fundierten Überblick über ihr TSM-Modell, dessen Historie, Entwicklung und Einsatz in der Psychodramatherapie sowie dessen Verbindung zur Neurobiologie und Bindungsforschung. Das TSM beinhaltet eine innere Rollenlandkarte für das strukturierte therapeutische Arbeiten, zunächst um wieder die Voraussetzungen für spontanes und kreatives Handeln zu schaffen, dann zur Integration von dissoziierten, traumatischen Affekten mit dem Ziel des posttraumatischen Wachstums. Durch den konsistenten und strukturierten Rahmen des TSM werden traumatische Erfahrungen in ihrer Bearbeitung in einem Toleranzfenster der Erträglichkeit und Belastung gehalten, so dass die ProtagonistInnen vor Retraumatisierung geschützt werden können. Das besondere an den Rollen ist hier, dass neben der Täter- und Opferrolle die Rolle der verlassenden Autorität inszeniert wird. In anschaulichen Fallbeispielen bringt die Autorin ihre Methode den LeserInnen berührend nahe.

Ein Beitrag zum Thema der potentiellen Auflösung der Subjekt-Objekt-Spaltung kommt von Jochen Becker-Ebel, der eine indische Perspektive in Form der Advaita Vedanta-Philosophie (vedische Lehren) zum Thema der Selbsterforschung und -erkenntnis einführt und mit den psychodramatischen Konzepten von Moreno verbindet. Das Ziel der Arbeit mit inneren Anteilen beschreibt er als einen Prozess, in dem zunächst eine Differenzierung zwischen dem Ich oder dem Innen und den Anderen oder dem Außen vollzogen wird, eine Disidentifikation von Selbstanteilen erfolgt, um dann wiederum die Identifikation mit dem Universum, dem Ganzen zu erfahren. Dabei geht es bei dem Konzept des Advaita um eine ungeteilte Einheit des Selbst mit dem großen Ganzen. Becker-Ebel verbindet dies mit dem psychodramatischen Konzept des Tele und sieht das Advaita quasi als dessen soziodramatische Erweiterung.

Der Artikel von Haseena Manipal, einer Transaktionsanalytikerin und Psychodramatikerin aus Indien, widmet sich den Gemeinsamkeiten und den Unterschieden dieser beiden Therapierichtungen. Dazu wird das klinische Material eines Fallbeispiels 
sowohl aus psychodramatischer als auch aus transaktionsanalytischer Perspektive interpretiert und so die theoretischen Konzepte praktisch veranschaulicht. Dadurch wird direkt und konkret deutlich, inwieweit beide Schulen Erkenntnisakzente setzen oder aber die gleichen Phänomene äquivalent erkennen, aber nur unterschiedlich benennen.

In der Rubrik Toolbox findet sich ein Beitrag von Maud Bermann und Hermann Küster, die zeigen, wie man innere Anteile mithilfe des Modells des inneren Teams psychodramatisch explorieren kann. Sie geben anhand von Fallbeispielen eine Anleitung zum strukturierten Erkunden von drei Anteilen des individuellen, inneren Teams in Form von schriftlichen Dialogen während einer Gruppenveranstaltung. Es werden hier gleichzeitig innere Anteile aller Gruppenmitglieder vertraulich ergründet und persönliche Entwicklungsthemen abgeleitet.

Der offene Teil beginnt mit einem Beitrag von Angelika Eisterer. Ihr Artikel beschreibt Problemlagen von Frauen, die von häuslicher Gewalt betroffen sind und stellt anhand eines Fallbeispiels Behandlungsansätze im monodramatischen Setting vor. Dabei geht es insbesondere um den differenzierten Einsatz der psychodramatischen Techniken des Doppelns und Spiegelns. Dabei wird angenommen, dass es zu einer Vermischung der Ich und Du-Grenzen während des traumatischen Erlebens, insbesondere bei Beziehungstraumatisierungen kommen kann. Dieser Beitrag ist im offenen Teil des Themenhefts platziert, hätte jedoch auch einen Platz im Thementeil finden können, da es inhaltlich deutlich um traumatisierte innere Anteile geht, die sich auch als „Selbstverunsicherung“ auswirken, ursächlich durch Übertragungen von Schuld und Verantwortung auf das Opfer entstanden sind.

In dem dritten englischsprachigen Beitrag des Heftes wird eine empirische Einzelfallstudie aus Südtirol von Dorothea Gstrein vorgestellt. Es geht hier um die Wirksamkeit von Psychodramagruppentherapie bei SchülerInnen mit einer Ängstlichkeit bezüglich des Unterrichtfachs Mathematik (Mathematics Anxiety). In der Studie werden quantitative und qualitative Methoden zur Demonstration der Wirksamkeit des Psychodramas in der Reduktion des Ausmaßes erlebter Belastung verwendet und anschaulich dargestellt. Es wird hierzu ein hermeneutisches Einzelfallstudiendesign zur Untersuchung der therapeutischen Wirksamkeit verwendet.

In ihrem Beitrag aus dem Bereich des Playbacktheaters stellt Jutta Heppekausen die szenische Bearbeitung von Diskriminierungserfahrungen dar. Hier geht es u.a. darum, wie der Aspekt der ,natürlichen Empathie“ mehr dem Mitfühlenden dienen kann als dass er einer Diskriminierungserfahrung, die im Playback dargestellt wird, gerecht werden kann. Heppekausen untermauert die Erfahrungen aus der Praxis, die sie anhand von Fallbeispielen veranschaulicht, mit differenzierten theoretischen Überlegungen aus der kritischen Psychologie.

Krzysztof Ciepliński und Joanna Karkut-Rzondkowska haben eine Gruppe von Studierenden beforscht, die ein dreitägiges Training mit erlebnisorientierten Methoden absolvierten. Ihre Studie widmet sich der Frage, welche Interventionen die TeilnehmerInnen für ihren Lernprozess als hilfreich bzw. als hinderlich erlebten. Die Rückmeldungen belegen einerseits, dass der Einsatz erlebnisaktivierender Methoden den Lernprozess in der Gruppe unterstützen können; auf der anderen Seite zeigen die zwischen den Gruppen, aber auch innerhalb der Gruppe zum Teil stark divergierenden Einschätzungen, dass solche Methoden mit Bedacht angewendet werden 
müssen und dass der erlebte Nutzen stark vom jeweils individuellen Gruppenprozess abhängt.

In der Rubrik ,Vernetzung“ findet sich die ins Deutsche übersetzte Fassung eines Interviews, das Jörg Jelden mit Ron Wiener zum Anlass von dessen letzter Lehrtätigkeit an der „Soziodrama Akademie“ in Berlin geführt hat.

Im Namen der gesamten ZPS-Redaktion wünschen wir Ihnen viel Freude beim Lesen.

Kristina Scheuffgen, Falko von Ameln 